\title{
A influência da actividade física na saúde mental positiva de idosos
}

\author{
H.M. Fernandes, J. Vasconcelos-Raposo, E. Pereira, J. Ramalho, S. Oliveira
}

\begin{abstract}
O presente estudo objectiva investigar os níveis de actividade física de adultos em idade avançada e a sua influência nos domínios da satisfação com a vida, auto-estima e crescimento pessoal. A amostra foi constituída por 168 indivíduos de ambos os sexos, com idades compreendidas entre os 60 e os 95 anos $(M=72.06, D P=6.83)$. Os resultados evidenciam que cerca de $41.1 \%$ da amostra é fisicamente inactiva, enquanto somente $31.5 \%$ dos idosos reporta níveis iguais ou superiores às recomendações internacionais de actividade física. As análises comparativas e correlacionais demonstram que um aumento dos níveis de prática de actividade física traduz-se em níveis superiores de satisfação com a vida, auto-estima e crescimento pessoal, sendo esse efeito superior nos idosos que praticam pelo menos 30 minutos de actividade física aeróbia de intensidade moderada durante cinco ou mais dias da semana.
\end{abstract}

Palavras-chave: actividade física, saúde mental positiva, idosos

\section{The influence of physical activity in the positive mental health of the elder}

This study aim to investigate the physical activity levels of older adults and its effect on life satisfaction, self-esteem and personal growth. A sample of 168 elderly persons of both sexes with ages between 60 and 95 years $(M=72.06, S D=6.83)$ was used. Results reveal that about $41.1 \%$ of the sample is physically inactive, while only $31.5 \%$ of the older adults reported physical activity levels equal or above international recommended amounts. Correlation and comparative analysis demonstrate that physical activity participation is associated with higher levels of life satisfaction, self-esteem and personal growth, and that this effect is superior in the elderly people who practice at least 30 minutes of moderate-intensity aerobic physical activity on five or more days a week.

Key words: physical activity, positive mental health, elderly

Submetido: 29.03.2009 | Aceite: 17.04.2009

Helder M. Fernandes. Doutor em Psicologia. Docente convidado da Universidade de Trás-os-Montes e Alto Douro. Membro integrado do CIDESD.

José Vasconcelos Raposo. Professor Catedrático em Psicologia da Universidade de Trás-os-Montes e Alto Douro. Membro integrado do CIDESD.

Estrela Pereira, Joana Ramalho e Sílvia Oliveira. Alunas da Licenciatura em Psicologia da Universidade de Trás-os-Montes e Alto Douro.

Endereço para correspondência: Universidade de Trás-os-Montes e Alto Douro, Rua Dr. Manuel Cardona, 5000-558 Vila Real. E-mail: hfernandes@utad.pt 
O envelhecimento das populações é um dos fenómenos que mais se evidencia nas sociedades actuais, pelo que esta situação resulta de um conjunto de avanços nas ciências biomédicas, tecnológicas e comportamentais, as quais se têm preocupado com o aumento do bem-estar e a qualidade de vida deste segmento populacional. Em Portugal, durante os últimos 50 anos, verificou-se um aumento notório do envelhecimento demográfico, definido pelo aumento da proporção das pessoas idosas na população total, em detrimento da população jovem e/ou da população em idade activa, traduzindo-se num incremento de $140 \%$ da população idosa no período entre 1960 e 2001 e constituindo cerca de $16.4 \%$ do total da população (Instituto Nacional de Estatística, 2002).

Enquanto processo (ou conjunto de processos) multifacetado que ocorre aos níveis molecular, celular e orgânico, o envelhecimento envolve alterações estruturais e funcionais em quase todo o organismo, originando evidentes manifestações ao nível psicomotor, socioafectivo e cognitivo. Estas mudanças ocorrem de modo assíncrono, pelo que para compreender o envelhecimento é necessário distinguir entre os seus processos primários e secundários (Berger, Pargman, \& Weinberg, 2007), que constituem eixos distintos, mas interactivos. Os processos primários estão intimamente associados com a idade cronológica e envolvem a perda "natural" da visão, do cabelo, as alterações na composição corporal, a desmineralização óssea e a diminuição da aptidão física. Por sua vez, os processos secundários incluem factores comportamentais e ambientais (e.g. stress elevado, consumo de tabaco, sedentarismo), assim como, a influência de doenças como a diabetes, artrite e o cancro. Implicitamente, assume-se que o estilo de vida de um indivíduo possa inferir importantes repercussões em ambos níveis dos processos de envelhecimento.

No âmbito das diversas áreas científicas que têm procurado desenvolver recursos que contribuam para o aumento da longevidade (ou qualidade de vida associada), destaca-se a linha de pesquisa sobre a influência da prática de actividade física na idade adulta avançada, que tem argumentado que o exercício físico é uma experiência necessária e fulcral na vida da maioria dos idosos.

A imensa evidência empírica existente até ao momento e revisada em diversas metaanálises (e.g. Netz, Wu, Becker, \& Tenenbaum, 2005; Reifschneider, 1998) demonstrou que a actividade física é um importante mecanismo comportamental para atenuar os efeitos degenerativos do envelhecimento nos domínios físico, social e mental, bem como, promover a independência funcional e inerente autonomia. De entre os benefícios reportados na literatura destaca-se que a prática de actividade física na idade adulta avançada está associada a uma melhoria da quantidade e qualidade do sono, a um padrão mais saudável de um conjunto de indicadores 
fisiológicos (consumo máximo de oxigénio, tensão arterial, peso corporal, densidade óssea, entre outros), a um menor risco de quedas tão usuais e nocivas neste período de vida, a um melhor funcionamento cognitivo, a maiores níveis de satisfação com a vida e auto-percepções e a uma menor prevalência e severidade dos sintomas de ansiedade e depressão, quer em populações clínicas, como não clínicas (Berger et al., 2007; Colcombe \& Kramer, 2003; Reifschneider, 1998; Schechtman \& Ory, 2001).

Perante o exposto, o presente estudo pretende contribuir com a apresentação da evidência do impacto da prática de actividade física na saúde mental de idosos, tendo em consideração um domínio pouco explorado nesta fase do curso da vida - a saúde mental positiva. Vários autores têm argumentado por alguma saliência na defesa e proposição deste domínio, pelo que os modelos resultantes têm-se centrado no pressuposto de que o bem-estar emocional é delimitado como algo mais que a mera ausência de sintomas psicopatológicos (Jahoda, 1958; Seligman, 2002; Ryff \& Singer, 1998). Neste âmbito, a investigação aqui retratada pretende respeitar estas premissas, procurando contribuir para a colmatação da reduzida pesquisa em idosos sobre este domínio, na medida em que a depressão tem sido a dimensão preferencialmente retratada pelos investigadores (Netz et al., 2005; Strawbridge, Deleger, Roberts, \& Kaplan, 2002; Söjsten \& Kivela, 2006; Reifschneider, 1998). Para além disto, insere-se numa linha de investigação que temos procurado estabelecer e desenvolver, centrada no estudo do domínio do bem-estar psicológico ao longo do continuum do desenvolvimento humano (Fernandes \& Vasconcelos-Raposo, 2008).

Durante muito tempo, as investigações no domínio do envelhecimento procuraram conceptualizar o significado de um envelhecimento bem sucedido na inclusão de dimensões como a felicidade, equilíbrio emocional, bem-estar subjectivo e depressão, permitindo consolidar algumas bases para se repensar e conceptualizar novas pesquisas centradas no funcionamento psicológico positivo na idade avançada (Ryff, 1989a). Objectivando a reorientação da discussão teórica e filosófica para uma concepção mais positiva do indivíduo, Ryff (1989a, 1989b) propôs um modelo multidimensional do bem-estar psicológico que integra seis dimensões geradas a partir da literatura subjacente às teorias do desenvolvimento, psicologia clínica e dos critérios de saúde mental positiva propostos por Marie Jahoda (Fernandes \& Vasconcelos-Raposo, 2008), a saber: domínio do meio, aceitação de si, relações positivas com os outros, autonomia, objectivos na vida e crescimento pessoal.

O crescimento pessoal constitui uma dimensão do modelo integral supracitado que representa o desenvolvimento contínuo do ser humano, portador da necessidade e, ao mesmo tempo, da capacidade de actualização e realização do seu potencial de crescimento e expansão como pessoa ao longo da sua vida, pelo que, para isso, o 
indivíduo tem de estar aberto a novas experiências (Ryff, 1989a, 1989b). No entanto, o processo de crescimento (leia-se desenvolvimento) pessoal pode ser afectado por sentimentos de estagnação, monotonia e falta de interesse na vida e também por confrontos com limitações decorrentes de um evento não esperado (ou aceite), pelo que o envelhecimento enquanto fenómeno contínuo abarca consideravelmente grande parte destas condições. Todavia, a capacidade de adaptação a qualquer mudança exige uma alteração pessoal contínua e, neste sentido, o envelhecimento satisfatório depende do equilíbrio entre as suas potencialidades e limitações (Ryff, 1989b). Keyes e Ryff (1998) consideram que o ser humano é portador de um desafio duplo de crescimento pessoal e de percepção das suas potencialidades, pelo que é na sequência desta superação ao longo da vida, que emerge um funcionamento psicológico positivo.

Relativamente às suas investigações iniciais, Carol Ryff (Ryff, 198gb; Ryff \& Keyes, 1995) demonstrou que o segmento idoso das suas amostras tendia a reportar menores níveis de crescimento pessoal em relação aos restantes grupos (jovens e adultos), sendo essas diferenças relativas às faixas etárias igualmente constatadas em estudos portugueses (Ferreira \& Simões, 1999; Novo, 2003; Novo, Duarte-Silva, \& Peralta, 1997). Como tal, importa perceber se o envolvimento na prática de actividade física contribui para um aumento dos níveis reportados de crescimento pessoal na população idosa.

Outro dos domínios intimamente associados ao bem-estar e muitas das vezes identificado como uma importante variável predictora é o das autopercepções (Fernandes \& Vasconcelos-Raposo, 2008). Na teoria e pesquisa contemporânea respeitante ao sentimento de si (self), o eu é entendido como um sistema dinâmico e multidimensional em que a informação acerca do self é organizada de acordo com o contexto envolvente e de acordo com as autopercepções específicas a cada domínio de vida (Harter, Waters, \& Whitesell, 1998; Oosterwegel \& Oppenheimer, 2002), pelo que o sentimento de si é deste modo mais correctamente descrito como um complexo sistema de construtos (Bernardo \& Matos, 2003).

Neste âmbito, a auto-estima é definida enquanto a dimensão avaliativa quantitativa do auto-conhecimento referente à forma como um indivíduo formula apreciações acerca de si próprio, quer seja acerca da sua auto-imagem, quer seja da sua prestação num dado domínio de vida (Rosenberg, 1965).

Perante as inúmeras contingências de vida e as transformações morfológicas e sociocognitivas associadas, a idade adulta avançada surge como um período de vida em que é capital o estudo das autopercepções, quer seja a um nível longitudinal deste período de vida (intra-ciclo), quer seja a um nível de enquadramento ao longo do 
curso de vida (inter-ciclo), pelo que estudos preliminares sugerem que a idade avançada (> 70 anos) constitui um dos períodos de vida em que se verificam maiores declínios nos níveis médios da auto-estima global (Robins, Tracy, Trzesniewski, Potter, \& Gosling, 2001).

Vários estudos têm sido efectuados com o propósito de analisar os efeitos do exercício físico nas autopercepções em global, e na auto-estima em específico, demonstrando que a prática de actividade física influencia positivamente a autoimagem e satisfação corporal, o auto-conceito e a auto-estima da população sénior (Beneddetti, Petroski, \& Gonçalves, 2003; Mazo, Cardoso, \& Aguiar, 2006; Rejeski \& Mihalko, 2001; Stathi, Fox, \& McKenna, 2002).

Com o propósito de apresentar uma explicação para o impacto do exercício na autoestima, Sonstroem e Morgan (1989) desenvolveram um modelo hierárquico representado de acordo com uma hierarquia (bottom-up), sendo que no topo se encontra a auto-estima e na sua base a auto-eficácia. Estes mesmos autores referem que as alterações nos níveis inferiores produzirão mudanças nos níveis superiores, isto é, na auto-estima. Desta forma, os sentimentos de auto-eficácia física vão se acumulando e generalizando, desenvolvendo sentimentos de competência. Estes dois últimos sentimentos, por sua vez, poderão influenciar os níveis de auto-estima directamente ou mediados pela aceitação física. Embora o propósito da presente investigação não seja o de validar esta proposição, consideramos que este modelo apresenta uma explicação plausível e coerente para a compreensão do impacto da actividade física na auto-estima dos idosos, dado a evidente redução da sua capacidade e competência motora e funcional.

Por sua vez, a satisfação com a vida pode ser definida como a dimensão cognitiva do modelo bem-estar subjectivo proposto por Diener (1984) e pressupõe que a avaliação global que uma pessoa faz da sua vida esteja relacionada com as percepções subjectivas (juízo cognitivo consciente) acerca da sua vida, pelo que esta apreciação também depende da comparação entre as circunstâncias de vida do indivíduo e do padrão por ele escolhido (Diener, Emmons, Larsen \& Griffin, 1985; Fernandes \& Vasconcelos-Raposo, 2008). Deste modo, a satisfação com a vida, abrange o estudo dos factores que constituem uma boa vida de acordo com os padrões que cada pessoa utiliza para definir o significado da sua vida (Diener, 2000).

Tendo em consideração a fase no (per)curso de vida que a idade adulta avançada representa, consideramos de todo interesse incluir esta dimensão no espectro de análise do presente estudo. Enquanto expressão subjectiva da reflexão sobre si num dado momento temporal (passado, presente ou futuro), a satisfação com a vida parece reflectir um passado de experiências, opções, decisões e comportamentos que 
"moldaram" o indivíduo, mas que também lhe concederam um know-how mais abrangente e consistente para lidar com as adversidades e exigências quotidianas, pelo que dados de investigações internacionais tendem a demonstrar que esta dimensão psicológica não diminui, necessariamente, com a avançar da idade (Diener \& Suh, 1998; Myers \& Diener, 1995).

A literatura trata de maneira algo semelhante os termos de envelhecimento bem sucedido, envelhecimento activo e qualidade de vida na velhice, sob o foco da satisfação com a vida (Jóia, Ruiz, \& Donalisio, 2007). Neste contexto, a ligação entre a satisfação com a vida, as funções cognitivas e a actividade física regular foi investigada em vários estudos que reportaram que a actividade física mantém ou aumenta as capacidades funcionais nos idosos e, neste sentido um dos factores que determina a satisfação com a vida no idoso é também o seu nível funcional de independência (Subas \& Havranb, 2004).

Tendo em consideração a imensa evidência empírica já existente sobre os benefícios da actividade física, bem como, as anteriores directrizes formuladas, um painel de autores sobre a alçada do ACSM (American College of Sports Medicine) e do AHA (American Heart Association) apresentou recentemente as novas recomendações de actividade física para a população idosa, sugerindo que estes deverão praticar um mínimo de 30 minutos de actividade física aeróbia de intensidade moderada durante cinco ou mais dias da semana, ou então, realizar um mínimo de 20 minutos de actividade física aeróbia de intensidade vigorosa em três dias da semana, podendo estas recomendações ser combinadas (Nelson, Rejeski, Blair, Duncan, Judge, King et al., 2007, p. 1439). Adicionalmente, os gerontes deverão participar em actividades de reforço muscular, flexibilidade e equilíbrio durante alguns dias da semana, salvaguardando necessárias adaptações à prescrição do exercício consoante as condições clínicas dos indivíduos.

Deste modo, constituem-se como objectivos específicos da presente investigação, os seguintes propósitos:

- Caracterizar os níveis de actividade física habitual auto-reportados; e,

- Analisar o efeito das variáveis actividade física, sexo e idade na saúde mental positiva de indivíduos idosos.

\section{Metodologia}

A presente investigação é de natureza transversal (cross-sectional) e do tipo causalcomparativo (ex post facto). 


\begin{abstract}
Amostra
A amostra global foi constituída por 168 indivíduos com idades compreendidas entre os 60 e os 95 anos (média: 72.06; desvio padrão: 6.83), residentes na região norte de Portugal (distrito de Vila Real). Ao nível do sexo, a amostra subdividiu-se em 102 mulheres $(60.7 \%)$ e 66 homens $(39.3 \%)$. Cerca de $47 \%(n=79)$ dos indivíduos tinha habilitação literária igual ou superior ao $4^{\circ}$ ano escolaridade ( $1^{\circ}$ ciclo do ensino básico). Somente 37 indivíduos (22\%) desempenhavam uma ocupação profissional remunerada na altura da recolha dos dados. A selecção da presente amostra orientou-se por um procedimento de amostragem probabilístico aleatório.
\end{abstract}

\title{
Instrumentos
}

A avaliação do nível de actividade física (AF) foi efectuada por método de autoresposta a duas questões que visavam quantificar os dias da última semana e de uma semana normal, em que os indivíduos realizavam pelo menos 30 minutos de actividade física moderada. Estas perguntas foram adaptadas da proposta de Prochaska, Sallis e Long (2001), sendo posteriormente calculado o valor final de actividade física habitual a partir da média das questões enunciadas. Para efeitos posteriores de análise, essa variável foi decomposta em dois construtos associados: (i) níveis de actividade física - o dias (inactivo - $\mathrm{G}_{1}$ ), 1 a 2 dias (insuficientemente activo G2), 3 a 4 dias (moderadamente activo - G3) e 5 ou mais dias (muito activo - G4); (ii) cumprimento das recomendações de actividade física - tendo em consideração as directrizes propostas por Nelson et al. (2007), a amostra foi dividida nos que não atingiam (menos de 5 dias) e nos que cumpriam ( 5 ou mais dias) as recomendações de actividade física moderada.

A avaliação da satisfação com a vida foi efectuada através de um instrumento de auto-resposta, especificamente desenvolvido por Diener et al. (1985) e traduzido e adaptado por Neto (1993) para a população portuguesa. Constituída por cinco itens, a SWLS - Satisfaction With Life Scale é respondida originalmente de acordo com uma escala tipo Likert de 7 pontos, indicando a concordância (ou não) com cada item apresentado. No presente estudo e por critérios de uniformidade em relação às outras escalas, optámos por uma escala de resposta de 5 pontos (1: discordo plenamente a 5 : concordo plenamente). Ao nível da consistência interna obteve-se um valor de 0.76 para o alpha de Cronbach.

Para o propósito da mensuração da auto-estima utilizámos a versão traduzida e adaptada por Santos e Maia (2003) da escala proposta por Rosenberg (1965) Rosenberg Self-Esteem Scale (RSES), que visa analisar a ponderação mais positiva ou negativa que os indivíduos fazem sobre as suas características pessoais. A RSES é constituída por 10 itens, sendo cinco de orientação positiva (e.g. "Globalmente, estou 
satisfeito(a) comigo próprio(a).") e cinco de orientação negativa (e.g. "Sinto que não tenho muito de que me orgulhar"). Embora no seu período inicial de desenvolvimento, este instrumento tenho sido respondido com base numa escala tipo Guttman, no presente estudo tratámos os itens de auto-estima de acordo com uma escala tipo Likert em que as opções de resposta variaram entre 1 (discordo plenamente) e 5 (concordo plenamente). Após a inversão dos 5 itens de orientação negativa foi possivel calcular um total da auto-estima, podendo os seus valores variar entre $10 \mathrm{e}$ 50. O valor de alpha de Cronbach obtido foi de 0.75 .

Para a análise do crescimento pessoal incluiu-se a escala correspondente, na forma de 9 itens da versão portuguesa de Novo et al. (1997), do questionário desenvolvido por Ryff (1989b) - EBEP: Escalas de Bem-Estar Psicológico (SPWB: Scales of Psychological Well-Being). Os itens foram seleccionados da forma longa das EBEP de acordo com recomendações de van Dierendonck (2005). Verificou-se um valor de consistência interna de 0.73 para este factor.

\section{Procedimentos}

Após uma informação inicial dos propósitos da investigação e a obtenção do consentimento informado por parte dos indivíduos, foram aplicados os questionários, sendo pedido para responderem de forma sincera e sendo thes garantindo o anonimato e a confidencialidade das suas respostas.

Após a inserção dos dados recolhidos numa matriz do SPSS v.15, procedeu-se a um conjunto de análises estatísticas. Inicialmente, efectuaram-se análises descritivas (frequências, média, desvio-padrão, assimetria e curtose) e de consistência interna das escalas. Com o intuito de medir a relação linear entre um conjunto de variáveis utilizámos o cálculo do $r$ de Pearson (também denominado de coeficiente produtomomento). Posteriormente, efectuaram-se análises uni (ANOVA) e multivariadas de variância (MANOVA) para determinar o efeito da prática de actividade física e do sexo nas dimensões psicológicas em estudo.

O nível de significância foi mantido em $5 \%$ ( $p<0.05)$.

\section{Resultados}

No quadro 1 são apresentados os parâmetros descritivos e de análise da normalidade de cada uma das escalas, bem como, o respectivo coeficiente de consistência interna. Considerando os resultados deste quadro constatou-se que os coeficientes de assimetria e achatamento situaram-se no intervalo da sua unidade, assumindo-se por isso a normalidade univariada da distribuição dos dados. 
Quadro 1. Análise descritiva e de consistência interna

\begin{tabular}{cccccc}
\hline & Média & $\begin{array}{c}\text { Desvio- } \\
\text { padrão }\end{array}$ & Assimetria & Curtose & $\begin{array}{c}\alpha \text { de } \\
\text { Cronbach }\end{array}$ \\
\hline Satisfação com a vida & 36.52 & 6.63 & 0.279 & 0.250 & 0.76 \\
Auto-estima & 17.04 & 4.38 & 0.442 & 0.171 & 0.75 \\
Crescimento pessoal & 27.81 & 6.34 & 0.387 & 0.163 & 0.73 \\
\hline
\end{tabular}

Relativamente aos níveis de AF habitual apresentados no quadro 2 verifica-se que cerca de $41.1 \%$ da amostra global é fisicamente inactiva.

Quadro 2. Frequências relativas (\%) da prevalência semanal da prática de actividade física

\begin{tabular}{lcccccccc}
\hline & Nunca & 1 dia & 2 dias & 3 dias & 4 dias & 5 dias & 6 dias & 7 dias \\
\hline $\begin{array}{l}\text { Amostra global } \\
\text { Sexo }\end{array}$ & 41.1 & 11.3 & 7.1 & 3.6 & 5.4 & 4.8 & 7.7 & 19.0 \\
$\quad \begin{array}{l}\text { Masculino } \\
\quad \text { Feminino }\end{array}$ & 31.8 & 9.1 & 9.1 & 4.5 & 7.6 & 1.5 & 10.6 & 25.8 \\
Faixa etária & 47.1 & 12.7 & 5.9 & 2.9 & 3.9 & 6.9 & 5.9 & 14.7 \\
6o-69 anos & & & & & & & & \\
$\begin{array}{l}\text { 70-79 anos } \\
\text { +80 anos }\end{array}$ & 28.8 & 15.2 & 7.6 & 4.5 & 9.1 & 7.6 & 7.6 & 19.7 \\
\hline
\end{tabular}

Em média, os idosos praticam $2.54 \pm 2.83$ dias por semana, observando-se uma superioridade $\left(F_{(1,166)}=4.98, p<0.05\right)$ reportada pelo sexo masculino em comparação com o feminino (3.14 \pm 2.92 dias vs $2.15 \pm 2.71$ dias). Quando analisado o cumprimento das recomendações internacionais de actividade física, somente $31.5 \% \quad(n=53)$ da amostra reportou níveis iguais ou superiores a 5 dias por semana, sendo mais uma vez esse valor superior no sexo masculino (37.9\% vs $27.5 \%)$. Quando diferenciados pela faixa etária, os níveis de actividade física suficiente para cumprir as recomendações diminuem com a idade (60-69 anos: 34.9\%; 70-79 anos: 30.0\%; e, mais de 80 anos: 28.6\%). Contudo, quando correlacionada a idade com o nível de AF não se verificou uma associação significativa $(r=-0.093, p>0.05)$.

Quando considerado o sexo da amostra em estudo, a MANOVA a um factor permitiu denotar um efeito significativo no conjunto das variáveis dependentes a um nível convencional $\quad\left(F_{(3,164)}=3.76, \quad p<0.05\right.$, Wilk's Lambda $\left.=0.936, \quad \eta^{2}=0.064\right)$, sendo apresentados os resultados no quadro 3 . 
42 | A influência da actividade física na saúde mental positiva de idosos

Quadro 3. Análise comparativa por sexo

\begin{tabular}{cccc}
\hline & Sexo Feminino & Sexo Masculino & \\
& $M \pm D P$ & $M \pm D P$ & $F$ \\
\hline Satisfação com a vida & $16.38 \pm 4.70$ & $18.06 \pm 3.62$ & $6.08 *$ \\
Auto-estima & $35.65 \pm 6.57$ & $37.88 \pm 6.53$ & $4.65^{*}$ \\
Crescimento pessoal & $26.77 \pm 6.22$ & $29.41 \pm 6.23$ & $7.19^{* *}$ \\
\hline
\end{tabular}

$* p<0.05, * * p<0.01, * * * p<0.001$

A análise do quadro 3 demonstra que o sexo masculino reportou níveis superiores e significativos $(p<0.05)$ em todas as dimensões em análise, quando comparado com o sexo feminino.

Por sua vez, quando correlacionada a idade com as dimensões psicológicas somente se constatou uma associação significativa com o crescimento pessoal $(r=-0.237$, $p<0.01)$, demonstrando que este tende a diminuir nesta fase do curso da vida.

Com o objectivo de clarificar a diferenciação da variável níveis de AF habitual relativamente às dimensões da saúde mental, efectuou-se uma análise de variância multivariada (MANOVA a um factor) que permitiu concluir que a mesma tem um peso significativo na diferenciação dos grupos, $F_{(9,394)}=4.75, p<0.001$, Wilk's Lambda=0.778, $\eta^{2}=0.080$, sendo apresentado no quadro 4 os valores das subsequentes análises univariadas.

Quadro 4. Análise comparativa por níveis de AF

\begin{tabular}{cccccc}
\hline & $\mathrm{G}_{1}(n=69)$ & $\mathrm{G}_{2}(n=31)$ & $\mathrm{G}_{3}(n=15)$ & $\mathrm{G}_{4}(n=53)$ & $F$ \\
& $M \pm D P$ & $M \pm D P$ & $M \pm D P$ & $M \pm D P$ & \\
\hline Satisfação com a vida & $15.54 \pm 4.68$ & $17.06 \pm 3.97$ & $16.20 \pm 2.15$ & $19.23 \pm 3.80$ & $8.27^{* * *}$ \\
Auto-estima & $33.94 \pm 6.57$ & $38.39 \pm 4.65$ & $38.07 \pm 6.14$ & $38.36 \pm 6.85$ & $6.53^{* * *}$ \\
Crescimento pessoal & $25.43 \pm 5.13$ & $28.71 \pm 6.75$ & $30.07 \pm 4.78$ & $29.74 \pm 6.98$ & $6.25^{* * *}$ \\
\hline
\end{tabular}

Nota: $G_{1}$ - inactivo, $\mathrm{G}_{2}$ - insuficientemente activo, $\mathrm{G}_{3}$ - moderadamente activo e $\mathrm{G}_{4}$ - muito activo $* p<0.05, * * p<0.01, * * * p<0.001$

No quadro anterior observa-se uma tendência crescente dos valores médios da satisfação com a vida, auto-estima e crescimento pessoal, sendo os valores mais baixos reportados pelos indivíduos inactivos. Os resultados da correlação corroboram esta evidência, na medida em que se verificaram associações positivas e significativas com as dimensões da saúde mental positiva analisadas, sugerindo que um aumento dos níveis de prática de actividade física traduz-se em níveis superiores de satisfação com a vida $(r=0.374 ; p<0.001)$, auto-estima $(r=0.308 ; p<0.001)$ e crescimento pessoal $(r=0.290 ; p<0.001)$. 
No que concerne o efeito do cumprimento dos níveis recomendados de $A F$, a MANOVA a um factor efectuada permite verificar que esta distinção de AF constitui um importante factor de diferenciação dos grupos ao nível das dimensões psicológicas $\left(F_{(3,164)}=8.72, p<0.001\right.$, Wilk's Lambda $\left.=0.863\right)$, sendo esse efeito bem superior aos anteriormente observados $\left(\eta^{2}=0.137\right)$.

Quadro 5. Análise comparativa por níveis de cumprimento das recomendações de AF

\begin{tabular}{cccc}
\hline & Não atinge & Atinge & \\
& $M \pm D P$ & $M \pm D P$ & $F$ \\
\hline Satisfação com a vida & $16.03 \pm 4.27$ & $19.23 \pm 3.80$ & $21.70 * * *$ \\
Auto-estima & $35.68 \pm 6.38$ & $38.36 \pm 6.85$ & $6.12 *$ \\
Crescimento pessoal & $26.92 \pm 5.83$ & $29.74 \pm 6.98$ & $7.43^{* *}$ \\
\hline
\end{tabular}

* $p<0.05, * * p<0.01, * * * p<0.001$

Através do quadro 5 é possível observar que os indivíduos idosos que atingem as recomendações da prática de exercício físico revelam níveis superiores de satisfação com a vida, auto-estima e crescimento pessoal, sendo essas diferenças estatisticamente significativas.

Em suma, os resultados do presente artigo sugerem que a prática de actividade física, preferencialmente de acordo com os níveis internacionais recomendados, traduz-se num perfil mais favorável do domínio da saúde mental positiva em idosos, traduzido nas dimensões da satisfação com a vida, auto-estima e crescimento pessoal.

\section{Discussão}

Os propósitos centrais desta investigação centraram-se na caracterização dos níveis auto-reportados de actividade física de uma população sénior e na compreensão do efeito desse envolvimento num conjunto de dimensões associadas à saúde mental positiva. Assim, mais do que procurar conhecer se a actividade física contribui para uma diminuição da prevalência e severidade de certas perturbações psiquiátricas em idosos, pretendeu-se analisar a influência da adopção de um estilo de vida activo em certas dimensões psicossociais, usualmente associadas à avaliação da saúde mental positiva.

Tanto quanto a literatura nos permite conhecer, o presente estudo alicerça-se na demanda de conhecimento numa área temática pouco explorada, da qual resulta uma escassa evidência teórico-empírica. Tal condição substancia-se no facto de que até ao momento não nos foi possível encontrar outra publicação que analisasse o efeito da actividade física na conjugação das três dimensões psicossociais referidas anteriormente. 
Estudos anteriormente publicados indicam que a prevalência de sedentarismo da população adulta portuguesa (mais de 18 anos) tende a oscilar entre os 6o e os 90\% (Martinez-Gonzalez, Varo, Santos, Irala, Gibney, Kearney et al., 2001; Sjöström, Oja, Hagströmer, Smith, \& Bauman, 2006), sendo esta uma das taxas mais elevadas da Europa (IEFS, 1999; Varo, Martínez-González, Irala-Estevéz, Kearney, Gibney, \& Martinez, 2003). Este dado é ainda mais preocupante quando se denota que o valor máximo da prevalência ocorre a partir dos 60 anos de idade, verificando-se uma ligeira superioridade por parte das mulheres - $91.5 \%$ vs $89.5 \%$ (Gal, Santos, \& Barros, 2005).

Relativamente à frequência da (in)actividade física praticada pela nossa amostra, os resultados indicam que $41.1 \%$ é fisicamente inactiva e cerca de $68.5 \%$ dos indivíduos não cumpre as recomendações mínimas internacionais. Pese embora a vasta reflexão e aparente divergência sobre a definição e classificação da inactividade física e sedentarismo com base nos pressupostos de dispêndio energético (Biddle, Gorely, Marshall, Murdey, \& Cameron, 2003; Murdey, Cameron, Biddle, Marshall, \& Gorely, 2004), os presentes dados sugerem que somente $31.5 \%$ destes adultos em idade avançada estão a participar em níveis de actividade física suficientes para auferir os benefícios para a saúde documentados na literatura (e.g. Netz et al., 2005; Reifschneider, 1998). Porém e quando confrontada esta taxa com as restantes prevalências acima referidas, observa-se um perfil mais activo por parte da presente amostra, embora as medidas de avaliação sejam distintas. Esta situação pode ser explicada, em parte, pelo facto de alguns elementos amostrais pertencerem a um grupo de praticantes de actividade física regular - os caminheiros de Vila Real - que já foram objecto de investigação anteriormente (Rodrigues \& Vasconcelos-Raposo, 2006).

No que concerne a distinção dos níveis de AF pelo sexo, o grupo dos homens tende a reportar valores médios mais elevados, assim como, uma taxa superior de cumprimento das recomendações internacionais, situação essa idêntica à retratada na literatura (Gal et al., 2005; Martinez-Gonzalez et al., 2001). Neste âmbito, as revisões existentes sobre a imensa evidência empírica publicada demonstram que o sexo, a par da idade, constituem os factores de influência da actividade física mais consistentes, em que os adultos mais novos e do sexo masculino tendem a evidenciar um estilo de vida mais activo (Sallis \& Owen, 1999; Trost, Owen, Bauman, Sallis, \& Brown, 2002). Várias explicações têm sido avançadas para a compreensão deste efeito a um nível individual, envolvendo influências genéticas, o (des)conhecimento dos benefícios do exercício, imposições sociais e baixa percepção de competência motora, embora outros factores contribuam para esta situação a diferentes níveis sociocontextuais. 
Quanto ao impacto da AF nas dimensões psicológicas, observaram-se influências positivas e significativas no que se refere quer aos distintos níveis de envolvimento, quer ao cumprimento das recomendações internacionais, evidenciando que o exercício físico é um mecanismo importantíssimo para a manutenção ou melhoria do bem-estar dos idosos, a par de outros benefícios de ordem biofisiológica já enunciados. Apesar de alguma especificidade (modo e tipo) da actividade física não ter sido contemplada neste estudo, os resultados obtidos sugerem que um aumento nos níveis de actividade física, se possível atingindo as recomendações, possibilita uma melhoria das percepções de contínuo desenvolvimento e enriquecimento pessoal (crescimento pessoal) e de atitude positiva de aceitação dos múltiplos aspectos do self e da vida passada, bem como, das boas e más qualidades pessoais (satisfação com a vida e auto-estima). Embora a natureza transversal da investigação não permita ignorar o efeito inverso (i.e., idosos com níveis de saúde mental positiva mais elevada podem participar mais facilmente em programas de AF), os presentes dados vão de encontro à evidência empírica que demonstra que a actividade física é um importante mecanismo comportamental para promover um envelhecimento mais saudável (Mazo et al., 2006; Netz et al., 2005; Reifschneider, 1998; Subas \& Havranb, 2004).

Esta influência da AF na saúde mental positiva dos idosos parece ser de extrema importância, dado que esta fase do curso da vida, usualmente se traduz por um declínio dos níveis de auto-estima (Robins et al., 2001) e crescimento pessoal (Ryff, 1989b; Ryff \& Keyes, 1995), com as inerentes repercussões no domínio da satisfação com a vida destes indivíduos. Deste modo, a prática da actividade física, enquanto elemento integral do estilo de vida de uma pessoa, parece incidir beneficamente nas alterações normais decorrentes do processo de envelhecimento, auxiliando na manutenção e melhoria de certas funções e contribuindo também para melhorar a sua dimensão psicossocial (Jost \& Santini, 2003; Ryff \& Singer, 1998; Vilela, Carvalho, \& Araújo, 2006).

A evidência empírica recente tem permitido a formulação de um conjunto de hipóteses e mecanismos desenvolvidos com o intuito de explicar o mais holisticamente possível a relação entre o exercício e saúde mental, abarcando efeitos aos níveis biofisiológico, social e mental. De entre estes, destaca-se o aumento da actividade hormonal, a promoção da "descarga" da tensão muscular, o incremento da temperatura corporal, a promoção de momentos de distracção/diversão, assim como, o desenvolvimento de percepções de mestria, de auto-controlo e de auto-eficácia (Leith, 1998). Embora a conclusão mais lógica e acertada, é de que muitos destes processos anteriormente enunciados ocorrem simultaneamente, originando assim uma combinação de efeitos indiciadores de melhoria nos estados de humor, 
consideramos que para a presente investigação, a proposta associada à auto-eficácia merece um maior destaque.

Desta forma, parece que as alterações na aptidão e aparência física decorrentes da prática de AF têm efeitos indirectos sobre as componentes da saúde mental positiva, com especial destaque para a auto-estima, tal como proposto pelo modelo de Sonstroem e Morgan (1989). Neste modelo, as mudanças da auto-eficácia associadas à actividade física indiciam alterações em subdomínios que contemplam a multidimensionalidade das medidas e que abarcam aspectos associados à satisfação corporal, ao desempenho físico, à autonomia funcional e às relações interpessoais, pelo que a evidência empírica mais recente oferece algum suporte para a confirmação destas assumpções (e.g. McAuley, Elavsky, Motl, Konopack, Hu, \& Marquez, 2005).

Pese embora os resultados obtidos, é necessário verificar-se alguma ponderação na sua interpretação, visto existirem algumas limitações metodológicas. O tamanho da amostra é reduzido e localizado regionalmente, pelo que a sua capacidade de generalização deve ser comedida. Para além disto, o carácter transversal da pesquisa não permite esclarecer a direcção (causal) do efeito entre actividade física e saúde mental positiva em idosos. Por fim, quer a medida utilizada para a mensuração da AF, quer os critérios definidos para a sua distinção por níveis, pecam pela inexistência de uma evidência empírica de suporte, sendo por isso necessários mais estudos centrados nestes pressupostos.

Deste modo, propomos que se aprofunde a compreensão da relação entre a AF e a saúde mental durante a idade adulta avançada, tendo por quadro de referência as inúmeras realidades sociais que se consubstanciam em distintos domínios de realização da vida dos idosos, tal como por exemplo, o seu local de residência (Paúl, Fonseca, Martín, \& Amado, 2003).

Em suma, o presente estudo demonstrou que cerca de $41.1 \%$ da amostra global é fisicamente inactiva, sendo estes níveis superiores no grupo feminino e mais velho dos idosos. Igualmente providenciou um conjunto de resultados que comprovam a importância da adopção de um estilo de vida activo por parte da população idosa, no que se refere à esfera positiva da saúde mental, pelo que o cumprimento das recomendações internacionais de AF surge como um patamar a atingir para a obtenção de níveis mais elevados de auto-estima, satisfação com a vida e crescimento pessoal.

Assim, consideramos que o exercício físico é um importante meio de promoção de saúde e de qualidade de vida, que fomenta benefícios ao nível físico, psíquico e social, permitindo aos idosos redescobrir novas e melhores formas de estar na vida de uma forma mais autónoma e independente. 


\section{Bibliografia}

Benedetti, T.B., Petroski, É.L., \& Gonçalves, L.T. (2003). Exercícios físicos, auto-imagem e auto-estima em idosos asilados. Revista Brasileira de Cineantropometria \& Desempenho Humano, 5(2), 69-74.

Berger, B.G., Pargman, D. \& Weinberg, R.S. (2007). Foundations of exercise psychology. Morgantown, WV: Fitness Information Technology.

Bernardo, R. \& Matos, M.G. (2003). Adaptação portuguesa do Physical Self-Perception Profile for Children and Youth e do Perceived Importance Profile for Children and Youth. Análise Psicológica, 21(2), 127-144.

Biddle, S.J.H., Gorely, T., Marshall, S.J., Murdey, I., \& Cameron, N. (2003). Physical activity and sedentary behaviors in youth: Issues and controversies. The Journal of the Royal Society for the Promotion of Health, 124(1), 29-33.

Colcombe, S. \& Kramer, A.F. (2003). Fitness effects on the cognitive function of older adults: A meta-analytic study. Psychological Science, 14, 125-130.

Diener, E. \& Suh, E. (1998). Age and subjective well-being: An international analysis. Annual Review of Gerontology and Geriatrics, 17, 304-324.

Diener, E. (1984). Subjective well-being. Psychological Bulletin, 95(3), 542-575.

Diener, E. (2000). Subjective well-being: The science of happiness and a proposal for a national index. American Psychologist, 55(1), 34-43.

Diener, E., Emmons, R.A., Larsen, R.J., \& Griffin, S. (1985). The satisfaction with life scale. Journal of Personality Assessment, 49(1), 71-75.

Fernandes, H. \& Vasconcelos-Raposo, J. (2008). O bem-estar psicológico em adolescentes: Uma abordagem centrada no florescimento humano. Vila Real: UTAD - CEDAFES Press.

Ferreira, J.A. \& Simões, A. (1999). Escalas de bem-estar psicológico (EBEP). In M.R. Simões, M.M. Gonçalves \& L.S. Almeida (Eds.), Testes e provas psicológicas em Portugal (vol. 2, pp. 111-121). Braga: APPORT/SHO.

Gal, D.L., Santos, A.C., \& Barros, H. (2005). Leisure-time versus full-day energy expenditure: A cross-sectional study of sedentarism in a Portuguese urban population. BioMed Central Public Health, 16(5). Obtido a partir de http://www.biomedcentral.com/1471-2458/5/16

Harter, S., Waters, P. \& Whitesell, N.R. (1998). Relational self-worth: Differences in perceived worth as a person across interpersonal contexts among adolescents. Child Development, $69(3), 756-766$.

IEFS (1999). A pan-EU survey on consumer attitudes to physical activity, body-weight and health. Luxembourg: Office for Official Publications of the European Communities.

Instituto Nacional de Estatística (2002). O envelhecimento em Portugal: Situação demográfica e socioeconómica recente das pessoas idosas. Revista de Estudos Demográficos, 32, 185208.

Jahoda, M. (1958). Current concepts of positive mental health. New York: Basic Books.

Jóia, L.M., Ruiz, T., \& Donalisio, M.R. (2007). Condições associadas ao grau de satisfação com a vida entre a população de idosos. Revista de Saúde Pública, 41(1), 131-138.

Jost, E. \& Santini, J. (2003). Benefícios da actividade física na saúde do idoso. Corpo em Movimento, 1(1), 103-115. 
48 | A influência da actividade física na saúde mental positiva de idosos

Keyes, C.L.M. \& Ryff, C.D. (1998). Generativity in adult lives: Social structural contours and quality of life consequences. In D.P. McAdams \& E.S. Aubin (Eds.), Generativity and adult development: How and why we care for the next generation (pp. 227-263). Washington, DC: American Psychological Association.

Leith, L.M. (1998). Exercising your way you better mental health: Fight depression and alleviate stress through exercise. Morgantown, WV: Fitness Information Technology.

Martinez-Gonzalez, M.A., Varo, J.J., Santos, J.L., Irala, J., Gibney, M., Kearney, J. et al. (2001). Prevalence of physical activity during leisure time in the European Union. Medicine and Science in Sports and Exercise, 33 (7), 1142-1146.

Mazo, G.Z., Cardoso, F.L., \& Aguiar, D.L. (2006). Programas de hidroginástica para idosos: motivação, auto-estima e auto-imagem. Revista Brasileira de Cineantropometria \& Desempenho Humano, 8(2), 67-72.

McAuley, E., Elavsky, S., Motl, R.W., Konopack, J. F., Hu, L., \& Marquez, D.X. (2005). Physical activity, self-efficacy, and self-esteem: Longitudinal relationships in older adults. Journal of Gerontology: Psychological Sciences, 6oB(5), 268-275.

Murdey, I.D., Cameron, N., Biddle, S.J.H., Marshall, S.J., \& Gorely, T. (2004). Pubertal development and sedentary behaviour during adolescence. Annals of Human Biology, 31(1), 75-86.

Myers, D.G. \& Diener, E. (1995). Who is happy? Psychological Science, 6(1), 10-19.

Nelson, M.E., Rejeski, W., Blair, S.N., Duncan, P.W., Judge, J.O., King, A.C. et al. (2007). Physical activity and public health in older adults: Recommendation from the American College of Sports Medicine and the American Heart Association. Medicine and Science in Sports and Exercise, 39, 1435-1445.

Neto, F. (1993). The satisfaction with life scale: Psychometrics properties in an adolescent sample. Journal of Youth and Adolescence, 22(2), 125-134.

Netz, Y., Wu, M., Becker, B., \& Tenenbaum, G. (2005). Physical activity and psychological wellbeing in advanced age: A meta-analysis of intervention studies. Psychology and Aging, 20(2), 272-284.

Novo, R.F. (2003). Para além da eudaimonia: O bem-estar psicológico em mulheres na idade adulta avançada. Coimbra: Fundação Calouste Gulbenkian/Fundação para a Ciência e a Tecnologia.

Novo, R.F., Duarte-Silva, E. \& Peralta, E. (1997). O bem-estar psicológico em adultos: Estudo das características psicométricas da versão portuguesa das escalas de C. Ryff. In M. Gonçalves, I. Ribeiro, S. Araújo, C. Machado, L.S. Almeida \& M. Simões (Eds.), Avaliação psicológica: Formas e contextos (vol. V, pp. 313-324). Braga: APPORT/SHO.

Oosterwegel, A. \& Oppenheimer, L. (2002). Jumping to awareness of conflict between selfrepresentations and its relation to psychological wellbeing. International Journal of Behavioral Development, 26(6), 548-555.

Paúl, C., Fonseca, A.M., Martín, I., \& Amado, J. (2003). Psychosocial profile of rural and urban elders in Portugal. European Journal of Psychology, 8(3) 189-197.

Prochaska, J.J., Sallis, J.F., \& Long, B. (2001). A physical activity screening measure for use with adolescents in primary care. Archives of Pediatric Adolescence Medicine, 155, 554-599. 
Reifschneider, E. (1998). Benefits of exercise for older adults: A meta-analysis. Dissertação de Doutoramento, Universidade de Wisconsin-Milwaukee.

Rejeski, W.J. \& Mihalko, S.L. (2001). Physical activity and quality of life in older adults. Journals of Gerontology, 56(11), 23-35.

Robins, R.W., Tracy, J.L., Trzesniewski, K., Potter, J., \& Gosling, S.D. (2001). Personality correlates of self-esteem. Journal of Research in Personality, 35, 463-482.

Rodrigues, A. \& Vasconcelos-Raposo, J. (2006). A relação entre o bem-estar psicológico, a autoestima, a depressão e a religiosidade entre praticantes de actividade física regular e não praticantes em idosos. Monografia não publicada, Vila Real: UTAD.

Rosenberg, M. (1965). Society and the adolescent self-image. Princeton: University Press.

Ryff, C.D. \& Keyes, C.L. (1995). The structure of psychological well-being revisited. Journal of Personality and Social Psychology, 69(4), 719-727.

Ryff, C.D. \& Singer, B. (1998). The role of the purpose in life and personal growth in positive human health. In P.S. Fry \& P.T.P. Wong (Eds.), The human quest for meaning: A handbook of psychology research and clinical applications (pp. 213-215). Mahwah, NJ:

Ryff, C.D. (1989a). Beyond Ponce de Leon and life satisfaction: New directions in quest of successful ageing. International Journal of Behavioral Development, 12(1), 35-55.

Ryff, C.D. (1989b). Happiness is everything, or is it? Explorations on the meaning of psychological. Journal of Personality and Social Psychology, 57(6), 1069-1081.

Sallis, J.F. \& Owen, N. (1999). Physical activity and behavioural medicine. Thousand Oaks: Sage Publications.

Santos, P.J. \& Maia, J. (2003). Análise factorial confirmatória e validação preliminar de uma versão portuguesa da escala de auto-estima de Rosenberg. Psicologia: Teoria, Investigação e Prática, 2, 253-268.

Schechtman, K.B. \& Ory, M.G. (2001). The effects of exercise on the quality of life of frail older adults: A preplanned meta-analysis of the FICSIT trials. Annals of Behavioral Medicine, 23(3), 186-197.

Seligman, M.E.P. (2002). Authentic happiness. New York: Free Press.

Sjöström, M., Oja, P., Hagströmer, M., Smith, B.J., \& Bauman, A. (2006). Health-enhancing physical activity across European Union countries: the Eurobarometer study. Journal of Public Health, 14, 291-300.

Söjsten, N. \& Kivela, S. (2006). The effects of physical exercise on depressive symptoms among the aged: A systematic review. International Journal of Geriatric Psychiatry, 21, 410-418.

Sonstroem, R.J. \& Morgan, W.P. (1989). Exercise and self-esteem: Rationale and model. Medicine and Science in Sports and Exercise, 21, 329-336.

Stathi, A., Fox, K.R., \& McKenna, J. (2002). Physical activity and dimensions of subjective wellbeing in older adults. Journal of Aging and Physical Activity, 10, 76-92.

Strawbridge, W.J., Deleger, S., Roberts, R.E., \& Kaplan, G.A. (2002). Physical activity reduces the risk of subsequent depression for older adults. American Journal of Epidemiology, 156, 328-334. 
$\mathbf{5 0}$ | A influência da actividade física na saúde mental positiva de idosos

Subas, F. \& Havranb, O. (2004). Evaluation of life satisfaction index of the elderly people living in nursing homes. Archives of Gerontology and Geriatrics, 41(1), 23-29.

Trost, S.G., Owen, N., Bauman, A.E., Sallis, J.F. \& Brown, W. (2002). Correlates of adult's participation in physical activity: Review and update. Medicine and Science in Sports and Exercise, 34(12), 1996-2001.

Van Dierendonck, D. (2005). The construct validity of Ryff's scales of psychological well-being and its extension with spiritual well-being. Personality and Individual Differences, 36, 629643.

Varo, J.J., Martínez-González, M.A., Irala-Estévez, J., Kearney, J., Gibney, M., \& Martínez, J.A. (2003). Distribution and determinants of sedentary lifestyles in the European Union. International Journal of Epidemiology, 32, 138-146.

Vilela, A., Carvalho, P. \& Araújo, R. (2006). Envelhecimento bem-sucedido: Representação de idosos, Revista.Saúde.Com, 2(2), 101-114. 